\title{
A NOTE ON THE PROPAGATION OF PLANE WAVES IN ELASTIC AND PLASTIC SOLIDS
}

\author{
G. J. COOPER and J. W. CRAGGS \\ (Received 1 September 1966)
}

\begin{abstract}
Some further numerical results are obtained for plane waves propagated into elastic and plastic solids. Quantitative agreement with experimental results indicates that rate of strain effects are not significant.
\end{abstract}

\section{Introduction}

In a recent article [1] a mathematical model for the propagation of waves in elastic solids was solved numerically for the case of a semi-infinite plate. Another article extended this treatment to plastic solids [2]. In both cases the differential equations were solved by standard finite difference techniques. The results indicated that the numerical techniques were not entirely satisfactory although it was believed that the gross behaviour obtained was correct.

It has since become possible to extend the investigations. Some simple refinements to the numerical techniques produce a marked improvement in the 'smoothness' of the final results and it has further been possible to obtain results at greater time intervals from impact. Results obtained for plastic solids now agree quantitatively with experimental results [3] and this suggests that rate of strain effects are not significant. The notation used is as in [2].

\section{Differential and difference equations}

We consider a plate of isotropic material bounded by $x_{2}= \pm a$, unbounded in $x_{3}$, and semi-infinite in $x_{1}\left(x_{1}, x_{2}, x_{3}\right.$ the Cartesian coordinates), with a constant force impact applied at $x_{1}=0$ in the direction of increasing $x_{1}$. In terms of the stress and strain tensors $\sigma_{i j}, \varepsilon_{i j}, i, j=1,2,3$, the dynamical equations can be expressed in scaled coordinates as

$$
2 \ddot{\varepsilon}_{i j}=\frac{\partial^{2} \sigma_{i k}}{\partial x_{j} \partial x_{k}}+\frac{\partial^{2} \sigma_{j k}}{\partial x_{j} \partial x_{k}}, \quad i, j, k=1,2,
$$

the superposed dots denoting double partial differentiation with respect to time, $t$. The initial and boundary conditions are given by 


$$
\begin{array}{ll}
\varepsilon_{i j}=\sigma_{i j}=0, i, j=1,2,3, & \text { for } t \leqq 0, x_{1}>0, \text { all } x_{2}, \\
\sigma_{11}=\sigma, \sigma_{12}=\sigma_{22}=0, & \text { for } t \geqq 0, x_{1}=0, \text { all } x_{2}, \\
\sigma_{12}=\sigma_{22}=0, & \text { for all } t, x_{1}, x_{2}= \pm a .
\end{array}
$$

As before we consider a mesh

$$
\begin{array}{ll}
t=l \delta t, & l=0,1, \cdots, \\
x_{1}=m \delta x_{1}, & m=0,1, \cdots, \\
x_{2}=n \delta x_{2}, & n=0,1, \cdots, N=\frac{a}{\delta x_{2}}
\end{array}
$$

where $\delta t, \delta x_{1}, \delta x_{2}$, are constant increments in $t, x_{1}, x_{2}$, and symmetry considerations allow us to deal only with non-negative values of $x_{2}$. The differential equations are now replaced by finite difference approximations, giving expressions similar to those used previously [2]. Again the expressions must be modified if $n=0, N$. The essential difference in numerical treatment lies in the removal of the restriction $\delta x_{1}=\delta x_{2}$.

If values $\sigma_{11}, \sigma_{22}, \sigma_{33}, \sigma_{12}, \varepsilon_{11}, \varepsilon_{12}$, are known for given values of $l, l-1$, for all $m>0$ and all $n$, then the difference expressions allow us to determine approximations to $\dot{\varepsilon}_{i j}[l, m, n]$ and hence to $\varepsilon_{i j}[l+1, m, n]$. Special starting procedures $(l=1)$ are not required.

\section{Stress strain relations}

In scaled coordinates the basic equations may be written in the form,

$$
\begin{aligned}
(1-2 v) \dot{\sigma}_{11}= & (1-\nu) \dot{\varepsilon}_{11}+\nu \dot{\varepsilon}_{22} \\
& +\left\{\sigma_{33} \frac{1+\nu}{3}\left(2 \sigma_{33}-\sigma_{11}-\sigma_{22}\right)-(1-v) \sigma_{11}-\nu \sigma_{22}\right\} G\left(\sigma_{i j}\right), \\
(1-2 \nu) \dot{\sigma}_{22}=\nu & \dot{\varepsilon}_{11}+(1-v) \dot{\varepsilon}_{22} \\
& +\left\{\sigma_{33}-\frac{1+v}{3}\left(2 \sigma_{33}-\sigma_{11}-\sigma_{22}\right)-\nu \sigma_{11}-(1-\nu) \sigma_{22}\right\} G\left(\sigma_{i j}\right), \\
\dot{\sigma}_{12}= & \dot{\varepsilon}_{12}-\sigma_{12} G\left(\sigma_{i j}\right), \\
\dot{\sigma}_{33}= & \nu\left(\dot{\sigma}_{11}+\dot{\sigma}_{22}\right)-\frac{1+\nu}{3}\left(2 \sigma_{33}-\sigma_{11}-\sigma_{22}\right) G\left(\sigma_{i j}\right) .
\end{aligned}
$$

Here $\nu$ is Poisson's ratio and $G\left(\sigma_{i j}\right)$ is a function, of position and time, which allows for work hardening.

These equations give approximate values for $\dot{\sigma}_{i j}[l, m, n]$ and hence for $\sigma_{i j}[l+1, m, n]$, once approximations have been obtained for $\dot{\varepsilon}_{i j}[l, m, n]$, as in the previous section. If $n=N$ we compute $\dot{\sigma}_{11}$ and hence $\dot{\sigma}_{33}$ (with $\dot{\sigma}_{22}$ set to zero). We then set 


$$
\sigma_{12}=\sigma_{22}=\varepsilon_{12}=0 .
$$

It is nevertheless convenient to also compute these values on the boundary, with $\sigma_{22}=0$ in the above equations, as a check on the adequacy of the numerical solution. This treatment of the boundary conditions differs from that described in the previous article $[2]$ when $G\left(\sigma_{i j}\right) \neq 0$. The present approach seems more natural but should not give significant differences.

Since $\varepsilon_{12}=0$ for $n=N$ there is some slight simplication in the corresponding difference approximation for $\dot{\varepsilon}_{\mathbf{1 2}}$.

We use a parabolic type of stress strain relation [2] and then the work hardening function is

$$
G\left(\sigma_{i j}\right)=\frac{F\left(\sigma_{i j}\right)}{2 k^{2}}\left\{1-\left(1-2 \theta k^{2}\right)^{\frac{3}{2}}\right\},
$$

where $\theta$ is a parameter and $F\left(\sigma_{i j}\right)$ is given by

$$
F\left(\sigma_{i j}\right)=\frac{1}{3}\left(2 \sigma_{11}-\sigma_{22}-\sigma_{33}\right) \dot{\varepsilon}_{11}+\frac{1}{3}\left(2 \sigma_{22}-\sigma_{11}-\sigma_{33}\right) \dot{\varepsilon}_{22}+2 \sigma_{12} \dot{\varepsilon}_{12} .
$$

Here the function $2 k^{2}$ is a function of time and position defined (and computed) by

$$
\begin{aligned}
w & =\frac{2}{3}\left[\sigma_{11}^{2}+\sigma_{22}^{2}+\sigma_{33}^{2}+3 \sigma_{12}^{2}-\sigma_{11} \sigma_{22}-\sigma_{22} \sigma_{33}-\sigma_{33} \sigma_{11}\right], \\
2 k^{2}(t) & =\max _{t^{\prime}<t}\left\{2 k^{2}\left(t^{\prime}\right), w(t)\right\} .
\end{aligned}
$$

The case $G\left(\sigma_{i j}\right)=0$ gives elastic behaviour and is used whenever unloading takes place [2].

The values of $\theta$ should be chosen so that $2 \theta k^{2} \leqq 1$ and this sets a maximum on the allowed values of $\theta$. An adequate bound seems difficult to obtain and the condition, $2 \theta k^{2} \leqq 1$, must be checked experimentally. Whenever $2 \theta k^{2}>1$ we set $1-2 \theta k^{2}=0$ in order to compute $G\left(\sigma_{i j}\right)$. The unloading criteria are not altered, nor is the value of $2 k^{2}$. This approach differs from that used before [2].

\section{Computation and results}

The equations were programmed in Algol for the Atlas computer at London University and some results obtained were compared with previous results $[1],[2]$. The agreement adequately verified the programming and the various modifications in the equations. The results now to be described were all obtained with $\sigma=\frac{1}{4}, N=8, v=\frac{1}{3}$.

We consider, first, elastic wave propagation. In figure 1 we have plotted the strain, $\varepsilon_{11}$, on the boundary, at half a plate width from the impact face, for increasing time. The solid line represents results obtained for $\eta_{1}=\frac{1}{8}, \eta_{2}=\frac{1}{2}$, and the dotted line gives results for $\eta_{1}=\eta_{2}=\frac{1}{2}$. We see that decreasing the ratio, 


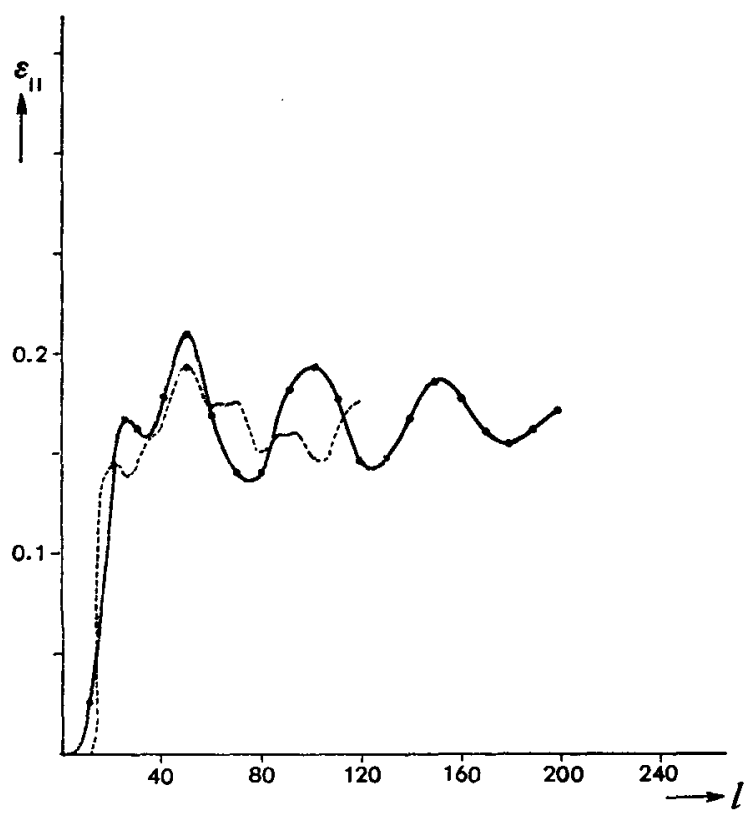

Figure 1

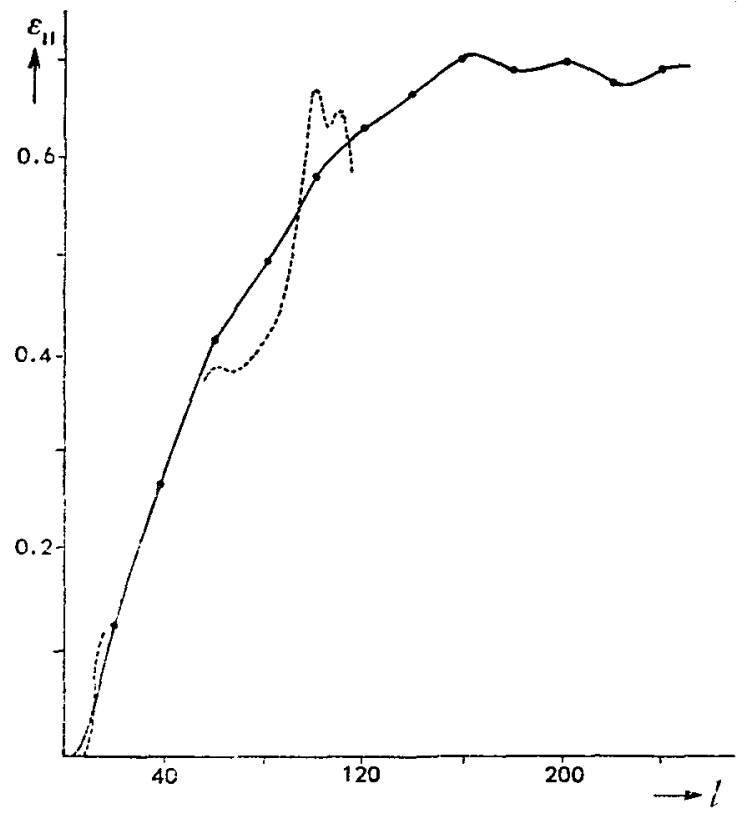

Figure 2 


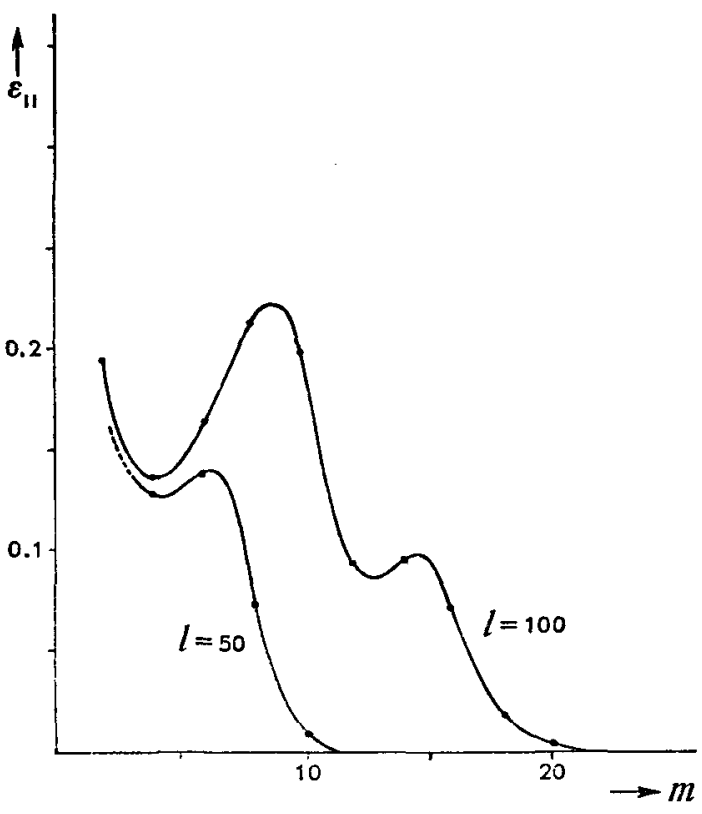

Figure 3

Table

Boundary points

$l=150, \quad N=8, \quad \theta=30, \quad \sigma=\frac{1}{4}, \quad v=\frac{1}{3}, \quad \eta_{1}=\frac{1}{8}, \quad \eta_{2}=\frac{1}{2}$

\begin{tabular}{rcrccccc}
\hline$m$ & \multicolumn{1}{c}{$\varepsilon_{11}$} & \multicolumn{1}{c}{$\varepsilon_{12}$} & \multicolumn{1}{c}{$\varepsilon_{22}$} & \multicolumn{1}{c}{$\sigma_{11}$} & \multicolumn{1}{c}{$\sigma_{22}$} & \multicolumn{1}{c}{$\sigma_{33}$} & $2 k^{2}$ \\
\hline 2 & 0.6896239 & -0.0000286 & -0.5996158 & 0.2579755 & 0.0006494 & 0.1264474 & 0.0332800 \\
4 & 0.4449181 & -0.0001023 & -0.3511708 & 0.2471886 & 0.0043663 & 0.1174638 & 0.0348931 \\
6 & 0.3598214 & 0.0002331 & -0.3128668 & 0.1744273 & -0.0119117 & 0.0901260 & 0.0362734 \\
8 & 0.3698522 & 0.0001298 & -0.2598031 & 0.2574452 & -0.0080262 & 0.1175309 & 0.0367853 \\
10 & 0.2546560 & -0.0001443 & -0.1552467 & 0.2271449 & 0.0091100 & 0.0975028 & 0.0259696 \\
12 & 0.1589159 & -0.0013656 & -0.1150172 & 0.1188353 & 0.0242410 & 0.0561394 & 0.0237503 \\
14 & 0.1376688 & 0.0007948 & -0.1223217 & 0.0819219 & -0.0181529 & 0.0418874 & 0.0217618 \\
16 & 0.1673920 & 0.0002024 & -0.0849370 & 0.1936962 & -0.0079967 & 0.0766002 & 0.0199915 \\
18 & 0.1040388 & -0.0002179 & -0.0360271 & 0.1559708 & 0.0080532 & 0.0559018 & 0.0124886 \\
20 & 0.0292571 & -0.0003426 & -0.0200858 & 0.0359143 & 0.0067728 & 0.0126255 & 0.0028159 \\
22 & 0.0325555 & 0.0002780 & -0.0259341 & 0.0374717 & -0.0032317 & 0.0129360 & 0.0022477 \\
24 & $\mathbf{0 . 0 3 9 6 8 3 1}$ & 0.0001052 & -0.0195121 & 0.0587892 & -0.0019993 & 0.0198762 & 0.0017885 \\
26 & 0.0190393 & -0.0000376 & -0.0070492 & 0.0309158 & 0.0004708 & 0.0103355 & 0.0004954 \\
28 & 0.0043234 & -0.0000273 & -0.0013342 & 0.0073112 & 0.0004004 & 0.0024374 & 0.0000277 \\
30 & 0.0005430 & -0.0000052 & -0.0001473 & 0.0009387 & 0.0000816 & 0.0003129 & 0.0000005 \\
\hline
\end{tabular}




$$
\frac{\eta_{1}}{\eta_{2}}=\frac{\delta x_{2}}{\delta x_{1}}, \quad \eta_{i}=\frac{\delta t}{\delta x_{i}}
$$

has smoothed the results markedly. Above $l=\mathbf{5 0}$ the strain is oscillating, with decreasing amplitude, about the known asymptotic value, $\frac{1}{6}$. Experiments indicate that increasing $N$, or further decreasing $\eta_{1} / \eta_{2}$, do not appreciably affect these results. The results for $\eta_{1}=\eta_{2}$ may be compared with those given in [2] (graph 1) and agreement is excellent, although the value of $N$ differs.

In [1] we plotted the strain, $\varepsilon_{11}$, for a fixed time interval and increasing $x_{1}$ for a constant velocity impact. Large oscillations away from the impact face indicated that some form of numerical instability might be present. It is not possible to obtain a direct comparison with present results but, in figure 3 , we have plotted the strain against increasing $x_{1}$, for $l=50$, 100 , and $\eta_{1}=\frac{1}{8}, \eta_{2}=\frac{1}{2}$. The behaviour is much smoother. Results are not markedly different for $\eta_{1}=\eta_{2}=\frac{1}{2}$ and this suggests that the instability previously observed was primarily due to the choice of too large a value of $\eta_{2}$.

Much improved results have also been obtained for plastic wave propagation. We have chosen $\theta=30$ since, with $\sigma=\frac{1}{4}$, this corresponds to the choice $\theta=120, \sigma=\frac{1}{8}$, in [2]. In figure 2 , the strain, $\varepsilon_{11}$, on the boundary at half a plate width from the impact face, has been plotted against increasing time. The solid line gives results for $\eta_{1}=\frac{1}{8}, \eta_{2}=\frac{1}{2}$, and the dotted line gives results for $\eta_{1}=\eta_{2}=\frac{1}{2}$. Again, decreasing the ratio $\eta_{2} / \eta_{1}$ has smoothed the results. The results for $\eta_{1}=\eta_{2}=\frac{1}{2}$ may be compared with those given in [2] (figure 2). There are some differences, due in part to the different choice of $N$ and possibly to the different treatment of boundary conditions. However, $2 \theta k^{2}$ has also been checked and these values increase markedly beyond unity, for points close to the impact face, and low values of $l$. The different approach employed in these circumstances has contributed appreciably to the observed differences in results.

The situation is considerably better for $\eta_{1}=\frac{1}{8}, \eta_{2}=\frac{1}{2}$. At half a plate width from the impact face the values of $2 \theta k^{2}$ increase to a maximum of about 1.02 but do not exceed unity until $l$ is greater than 100. Further from the impact face the values increase somewhat further and then decrease. This behaviour is probably caused by the use of finite step lengths and can presumably be completely overcome by decreasing $\eta_{1}$ and $\eta_{2}$ still further, or by increasing $N$. We have obtained some results for $N=12$ which suggest that this is so. The use of the stress strain relation with a non-horizontal asymptote would also prevent the trouble.

We observe that for $N=\mathbf{8}$ the strain rises to a plateau in accordance 
with experimental observations [3] and levels off at about $l=160$. For a plate of width one inch, and a material such as aluminium, $\delta t \simeq 0.18 \mu \mathrm{s}$ (for $\eta_{2}=\frac{1}{2}$ ). That is, the strain levels off at about $30 \mu \mathrm{s}$. This corresponds remarkably well with experimental results [3]. The table gives some results on the boundary for $l=150$. We should have $\sigma_{22}=\varepsilon_{12}=0$ and the values in these columns are indeed small.

\section{Errata}

Dr A. R. Curtis (A. E. R. E.) kindly pointed out a mistake in the article on elastic wave propagation [1]. Equation (13) should read

$$
\begin{aligned}
u_{j+1, k, l}= & -u_{j-1, k, l}+u_{j, k+1, l}+u_{j, k-1, l} \\
& +\frac{1}{2}\left(\frac{\delta t}{\delta x}\right)^{2}\left[u_{j, k, l+1}+u_{j, k, l-1}-u_{j, k+1, l}-u_{j, k-1, l}\right] \\
& +\frac{1}{8(1-2 v)}\left(\frac{\delta t}{\delta x}\right)^{2}\left[v_{j, k+1, l+1}-v_{j, k+1, l-1}-v_{j, k-1, l+1}+v_{j, k-1, l-1}\right] \\
& +0(\delta x)^{4},
\end{aligned}
$$

and there is a corresponding alteration in the equation for $\boldsymbol{u}_{j+1, k, 0}$. He also drew our attention to the likelihood of the presence of instability for large $x$.

\section{Acknowledgement}

We would like to thank Mrs A. Mayho who programmed the equations.

\section{References}

[1] G. J. Cooper and J. W. Craggs, 'Propagation of elastic waves', J. A ust. Math. Soc., 6 (1966) $55-64$.

[2] G. J. Cooper and J. W. Craggs, 'The propagation of plane waves in plastic solids', J. Aust. Math. Soc., 5 (1965), 349-364.

[3] J. F. Bell, J. App. Physics, 'Propagation of plastic waves in solids', 30, (1959) 196-201.

Institute of Computer Science

University of London 